\title{
Learning-Related Development of Context-Specific Neuronal Responses to Places and Events: The Hippocampal Role in Context Processing
}

\author{
David M. Smith and Sheri J. Y. Mizumori \\ Department of Psychology, University of Washington, Seattle, Washington 98195
}

\begin{abstract}
Contextual information plays a key role in learning and memory. Learned information becomes associated with the context such that the context can cue the relevant memories and behaviors. An extensive literature involving experimental brain lesions has implicated the hippocampus in context processing. However, the neurophysiological mechanisms of context coding are not known. Although "context" has typically been defined in terms of the background cues, recent studies indicate that hippocampal neurons are sensitive to subtle changes in task demands, even in an unchanging environment. Thus, the context may also include non-environmental features of a learning situation. In the present study, hippocampal neuronal activity was recorded while rats learned to approach different reward locations in two contexts. Because all of the training took place in the same environment, the contexts were defined by the task demands rather than by environmental stimuli. Learning to differentiate two such contexts was associated with the development of highly contextspecific neuronal firing patterns. These included different place fields in pyramidal neurons and different event (e.g., reward) responses in pyramidal and interneurons. The differential firing patterns did not develop in a control condition that did not involve a context manipulation. The context-specific firing patterns could modulate activity in extrahippocampal structures to prime context-appropriate behavioral responses and memories. These results provide direct support for a context processing role of the hippocampus and suggest that the hippocampus contributes contextual representations to episodic memories.
\end{abstract}

Key words: hippocampus; context; learning; place cell; interneuron; episodic memory

\section{Introduction}

Contextual information plays a key role in learning and memory. Learned information becomes associated with the context such that recall is best when testing takes place in the learning context (Godden and Baddely, 1975). The context primes relevant memories and serves as a cue that elicits conditioned responses (Blanchard and Blanchard, 1972; Balaz et al., 1980; Fanselow, 1986). An extensive literature involving brain lesions has implicated the hippocampus in context processing (for review, see Myers and Gluck, 1994; Anagnostaras et al., 2001; Maren, 2001). For example, hippocampal lesions impair conditioned fear responses to contextual stimuli (Kim and Fanselow, 1992; Phillips and LeDoux, 1992), and lesions of the hippocampus or entorhinal cortex render subjects insensitive to changes in the context (Penick and Solomon, 1991; Freeman et al., 1997). Also, subjects with fornix lesions were severely impaired in learning two different discrimination tasks that were trained in different contexts (Smith et al., 2004). In the same subjects, context-specific neuro-

Received Aug. 3, 2005; revised Dec. 22, 2005; accepted Jan. 30, 2006.

This work was supported by National Institutes of Health Grants MH67399 (D.M.S.) and MH58755 (S.J.Y.M.). We thank the following individuals for their comments during the preparation of this manuscript: I. Bernstein, J. Kim, J. H. Freeman Jr., and R. Swain.

Correspondence should be addressed to Dr. David M. Smith, Department of Psychology, Campus Box 351525, University of Washington, Seattle, WA 98195. E-mail: dmsmith4@u.washington.edu.

D01:10.1523/JNEUROSCI.3234-05.2006

Copyright $\odot 2006$ Society for Neuroscience $\quad$ 0270-6474/06/263154-10\$15.00/0 nal firing patterns were degraded in structures receiving hippocampal input via the fornix (anterior thalamus and cingulate cortex). These findings suggested that the hippocampus generates a unique context code that modulates processing in downstream structures. However, the neurophysiological mechanisms of context coding are not known.

Previous studies have defined "context" in terms of the static background cues. However, recent findings indicate that subtle changes in task demands are associated with remarkable alterations of the spatial firing patterns [i.e., place fields (O'Keefe and Dostrovsky, 1971)] of hippocampal pyramidal neurons, although the spatial environment was unchanged (Markus et al., 1995; Skaggs and McNaughton, 1998; Wood et al., 2000; Song et al., 2005). To the extent that such spatial firing is part of a context code (Nadel et al., 1985; Mizumori et al., 1999), these results suggest that hippocampal neurons differentially encode contexts that differ only in terms of the situational demands. Therefore, any definition of context, as it relates to hippocampal function, should also incorporate non-environmental task features.

Thus far, the relevance of these firing patterns to contextual learning has not been demonstrated. The present study was designed to explicitly test whether context-specific firing patterns develop when subjects learn to discriminate contexts. Hippocampal neurons were recorded while rats learned to retrieve rewards from two different locations in the same environment. Thus, the two contexts were defined by the reward location rather 
than by the environment. Each context was a distinct situation that required subjects to remember and approach a different reward location. If hippocampal neurons are involved in context processing, then context-specific neuronal responses should develop only when subjects learn to discriminate the two contexts and not in a control condition that does not involve a context manipulation.

Hippocampal interneurons are widely thought to code egocentric movement information (Feder and Ranck, 1973). It is not known whether these neurons play a role in context processing. However, interneurons respond to task-relevant stimuli (Wiebe and Staubli, 2001), suggesting an involvement in the mnemonic functions of the hippocampus. Thus, interneuron responses were also examined to determine whether context-specific firing develops during learning.

\section{Materials and Methods}

Subjects and surgical procedures. The subjects were 14 food-restricted ( $80-85 \%$ of free-feeding weight) adult male Long-Evans rats (Simonsen Laboratories, Gilroy, CA). Movable stereotrode recording electrodes, fabricated by twisting together two $25 \mu \mathrm{m}$ lacquer-coated tungsten wires (McNaughton et al., 1983a), were stereotaxically positioned just above the CA1 field of the hippocampus (2.5-4.5 mm posterior to bregma, 2.5 $\mathrm{mm}$ lateral, and $1.7 \mathrm{~mm}$ ventral). The rats were anesthetized with sodium pentobarbital $(40 \mathrm{mg} / \mathrm{kg})$. They were also given atropine sulfate $(0.2$ $\mathrm{mg} / \mathrm{kg}$ ) to prevent respiratory congestion, an antibiotic $(5 \mathrm{mg} / \mathrm{kg}$ Baytril), and an analgesic ( $5 \mathrm{mg} / \mathrm{kg}$ Ketofen). All procedures complied with guidelines established by the University of Washington Animal Care and Use Committee.

Behavioral training. It was reasoned that if the rats were required to perform different responses in two contexts that contained few distinguishing features, they would be forced to rely on internal context representations. Therefore, rats were trained to retrieve rewards (two drops of chocolate milk) from one location on a plus maze during the first half of each training session and from a different location in the same environment during the second half of the sessions. The two session halves constituted separate contexts, which were defined by the task demands rather than the background stimuli. The environment and the specific motor behaviors (e.g., locomotion, right and left turns) were equivalent across contexts. Thus, any differential firing patterns in the two contexts cannot be attributed to these factors.

The maze occupied a circular area, enclosed by curtains, with objects placed around the perimeter to serve as visual cues (for details, see Yeshenko et al., 2004). Trials began when the rats were placed on the maze facing outward at the end of an arm and ended when the rat arrived at the reward. During an intertrial interval (ITI) of $\sim 60 \mathrm{~s}$, the rats were placed on a platform adjacent to the maze. The position of the ITI platform was constant throughout training.

Before beginning regular training sessions, the rats were given a preliminary training session during which baseline neuronal and behavioral data were collected. During this session, the rats started each trial on a randomly designated arm and searched for rewards located on a different randomly designated arm. The rats were given two blocks of 10 training trials, separated by $30 \mathrm{~s}$ of darkness. The training procedures and behavioral requirements did not differ between the two blocks of trials. These random reward sessions served as a control condition that did not have a context manipulation.

After the random reward session, the rats were given daily training sessions consisting of two blocks of 15 trials each. During the first block of every training session, the reward was always placed at the end of the east arm. During the second block, the reward was always placed at the end of the west arm. The start positions for each trial were randomly designated from among the three nonreward arms. The two blocks were separated by $30 \mathrm{~s}$ of darkness to cue the rats that the second block was about to begin. Training continued with the same two reward locations presented each day until the rats attained a behavioral criterion of at least $75 \%$ correct choices. After achieving this criterion, the rats were given
2-10 additional training sessions for the collection of neuronal data during asymptotic performance.

Data collection and analysis. Neuronal spike data and video data were collected with the Cheetah Data Acquisition System (Neuralynx, Tucson, AZ). Before training, the recording probes were lowered in $40 \mu \mathrm{m}$ increments until isolatable units were encountered. The electrodes were advanced to obtain new units when records were lost. The electrodes were also advanced to obtain new units between asymptotic performance sessions to improve the yield. Signals from the electrodes were amplified $3000-10,000$ times, filtered at $600 \mathrm{~Hz}$ and $6 \mathrm{kHz}$, and digitized. All waveforms exceeding a user-defined threshold were stored to disk along with their time of occurrence for off-line analysis. Standard spike-sorting techniques were used to separate the multiunit records into component single units (MClust; A. D. Redish, University of Minnesota, Minneapolis, $\mathrm{MN}$ ). Waveform features used for sorting included spike amplitude, spike width, waveform principle components, and waveform area. Additional template-matching algorithms were used to facilitate sorting. The rat's position and direction of travel were monitored by digitized video (sampled at $20 \mathrm{~Hz}$ ) of a light-emitting diode array attached to the rats head. Video data were also used to establish the time of the trial start, arrival at the reward, and return to the ITI platform after each trial.

Units with spike widths $>270 \mu$ s were classified as pyramidal neurons, and units with spike widths $<270 \mu$ s were classified as interneurons. An additional firing frequency criteria $(>3 \mathrm{~Hz})$ was established to help exclude pyramidal neurons, which are known to exhibit event-related responses, from the analyses of interneuron event responses. The mean spike widths and firing frequencies were $328 \mu \mathrm{s}$ and $2.2 \mathrm{~Hz}$ for pyramidal neurons and $176 \mu$ s and $15.0 \mathrm{~Hz}$ for interneurons.

To examine the spatial firing patterns of the neurons, the firing frequencies (number of spikes divided by time in the pixel) were binned into pixels $(2.8 \times 2.8 \mathrm{~cm})$ according to the position of the rat. The firing frequency data were smoothed by replacing the frequency in each pixel with the weighted average of the $3 \times 3$ array of pixels in which it was centered.

Pyramidal neurons were classified as having a place field if they (1) fired in at least four adjacent pixels but less than half of the maze area, (2) had a within-field firing rate at least twice that of the firing rate outside the field, and (3) fired during $>50 \%$ of the passes through the field. To assess event-related firing, perievent time histograms (100 ms bins) were constructed with the data centered on the arrival at the reward location, the start of training trials, and the return to the ITI platform after training trials. Separate histograms were constructed for the two blocks of trials within each session. The firing frequencies of the 10 preevent bins were compared with the 10 postevent bins using the Wilcoxon signed rank test. Neurons with a significant $(p<0.05)$ difference between preevent and postevent firing rates were classified as having an event response.

The firing of many hippocampal interneurons is correlated with the rats' velocity such that apparent event responses could be attributable to changes in locomotion occurring at the time of events (e.g., trial start or arrival at the reward). To eliminate this possibility, interneurons $(n=25)$ with velocity-related firing that could have accounted for observed event responses were excluded from the analyses.

The data of all of the neurons that exhibited event responses were further analyzed to detect differences in event-related neuronal firing across the two blocks of each training session. For these analyses, the values in the histograms were normalized ( $z$-transformation) using the mean and SD of the firing rate recorded for $5 \mathrm{~s}$ before the event. Thus, the firing rate data were expressed in standard units of change from the preevent baseline. The 10 postevent bins ( $1 \mathrm{~s}$ ) of the first block were then compared with 10 postevent bins of the second block.

After classification of the neurons with regard to their responses, the data were analyzed to determine whether context-specific firing developed during learning. In the case of neurons that had place fields, separate firing rate maps were constructed for the first and second blocks of each training session, and a pixel by pixel correlation coefficient (Pearson's $r$ ) of the firing rates was computed. Only the pixels visited in both blocks were used. The correlation coefficients served as a measure of the similarity of the spatial firing patterns across the two blocks of trials. The correlation coefficients of neurons recorded during the random reward 
session were compared with the coefficients of neurons recorded during asymptotic performance with ANOVA. To assess the development of context-specific event responses, the numbers of neurons with event responses that differed across the two blocks during the random reward session and during asymptotic performance were submitted to $\chi^{2}$ analysis.

\section{Results}

\section{Behavior}

The rats achieved the performance criterion in an average of 6.7 sessions. During postcriterial asymptotic performance sessions, the rats correctly chose the appropriate goal arm (with no erroneous entries onto nonrewarded arms) on $79.1 \%$ of the trials. This level of performance was significantly greater than chance performance of $33.3 \%\left(t_{(13)}=33.54 ; p<0.001\right)$. Performance during the random reward session did not differ from chance $\left(35.7 \%\right.$ correct; $\left.t_{(13)}=1.01 ; p<0.35\right)$.

\section{Spatial firing patterns}

Hippocampal pyramidal neurons developed highly differentiated spatial firing patterns in the two contexts (Fig. 1). Four hundred eleven pyramidal neurons were recorded, 105 during the random reward session and 306 during asymptotic performance. Thirty four and 120 of the neurons recorded during the random reward session and asymptotic performance, respectively, exhibited place fields. Pixel by pixel correlations reflecting the similarity of the spatial firing patterns across the two blocks of the training session were submitted to ANOVA, which indicated that the correlations were significantly reduced during asymptotic performance relative to the random reward session $\left(F_{(1,152)}=7.51\right.$; $p<0.01)$. Thus, spatial firing became more distinct in the two contexts after learning.

Some neurons $(n=11)$ exhibited place fields that were dependent on complex conjunctions of events, such as a place field that was exhibited only when the rat arrived at the field from a particular start arm. It is not clear whether these place fields were controlled by the subject's current position relative to a particular start and goal location (i.e., relational coding), the specific trajectory or path taken by the subject (trajectory coding), or whether they fired in relation to previous or intended future actions (prospective and retrospective coding) (Frank et al., 2000; Wood et al., 2000; Ferbinteanu and Shapiro, 2003). In any case, the current results indicated that even these complex firing patterns were specific to a particular context. Figure 2 illustrates neuronal firing in response to the conjunction of a location and start position in one context and a different location and start position in the other context. A second example is illustrated in Figure $5 B$, in which the neuron reliably fired in response to the reward whenever the rat had come from the west start arm. This firing was present in one context but not the other. Nine neurons exhibited context-specific responses that depended on complex conjunctions of places and events. Two neurons exhibited complex responses that did not differ across contexts.

Because the rats of the present study approached different reward locations, the paths they took and their direction of travel differed in the two contexts. Both the direction of travel and the specific path followed by the rats have been shown to influence the spatial firing of hippocampal neurons (McNaughton et al., 1983b; Frank et al., 2000). Most of the spatial and event responses observed here were not specific to a particular start position and therefore could not have been modulated by the path of the rat. However, as described above, some neurons did fire only when the rat arrived at the field from a specific start position. The fact that these neurons could exhibit different start-specific responses in the two contexts (Fig. 2) suggests that the responses were modulated by the context rather than the path taken by the rat.

Possible directional influences were examined by comparing neuronal firing during passes through the fields in one context to firing during passes through the same region in the same direction in the other context. In cases involving 110 of the 120 neurons (139 place fields), the rats traversed the fields in the same direction in both contexts, because either the field was on a part of the maze that was traversed in the same direction in both contexts (e.g., the north or south arms) or the rat made errors in one context that involved passing through the field in the same direction as the other context. During asymptotic performance, the firing frequency was significantly greater during passes through the fields in one context than during passes through the same region in the same direction in the other context (paired $t$ test, $\left.t_{(138)}=9.98 ; p<0.001\right)$. Thus, the spatial firing was highly differentiated in the two contexts even when the analysis controlled for the rat's direction of travel. Consistent with this, recent findings from our laboratory indicate that hippocampal neurons exhibit context-specific firing, depending on the problem-solving strategy, even when the rats traversed the same path in the same direction (Yeshenko et al., 2001).

Studies have shown that a reward can serve as a particularly salient orienting cue and that moving the reward location causes place fields to migrate toward the new reward location (Breese et al., 1989; Hollup et al., 2001; Kobayashi et al., 2003) (but see Speakman and O'Keefe, 1990). Other studies have shown that place fields can exhibit "path equivalence," wherein they fire in the same relative location along a path (e.g., inbound run, right turn, outbound run), even when the paths do not traverse the same region of space (Frank et al., 2000). In the present study, the equivalent paths are $180^{\circ}$ apart (e.g., the right turns toward the east arm are $180^{\circ}$ from the right turns toward the west arm). Thus, both of these explanations (reorienting and path equivalence) would predict that the place fields recorded in the two contexts would be $180^{\circ}$ apart. Analysis of the cross-block correlations computed after rotating the data of the second block $180^{\circ}$ indicated that, rather than improving the correlations, as would be expected if the fields had reoriented to the new reward location or followed equivalent paths, the correlations decreased significantly after the rotation (paired $t$ test, $t_{(134)}=7.23 ; p<0.01$ ). In a small number of cases ( 9 of the 120 neurons with place fields), the correlation scores improved substantially (i.e., by $>0.2$ ) after the rotation. However, it is not clear whether these cases represent realignment of the place fields or whether the fields were $\sim 180^{\circ}$ apart in the two contexts simply as a result of chance.

\section{Event-related responses of pyramidal neurons}

Pyramidal neurons developed robust context-specific responses to task-relevant stimuli and events, including the reward, the start of training trials, and the arrival at the ITI platform after trials (Fig. 3). The numbers and percentages of neurons that exhibited various kinds of responses are given in Table 1. The percentage of neurons exhibiting a context-specific reward response increased significantly after training $\left(\chi_{(1)}^{2}=12.68 ; p<0.001\right)$. Similar training-related increases were found in the percentage of neurons with context-specific ITI responses $\left(\chi_{(1)}^{2}=7.79 ; p<0.01\right)$. Neurons also exhibited context-specific trial start responses. However, the analyses did not detect a training-related change in the percentage of neurons with these responses (5.2\% during asymptotic performance compared with $2.9 \%$ during the random reward session; $\left.\chi_{(1)}^{2}=0.93 ; p<0.34\right)$. 
Random Reward Block 1
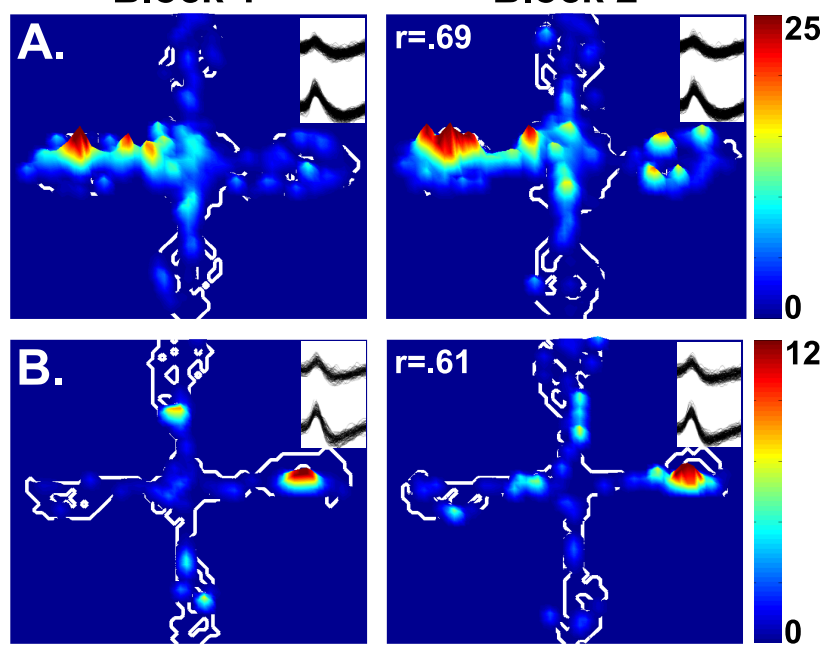

Asymptotic Performance

Context A
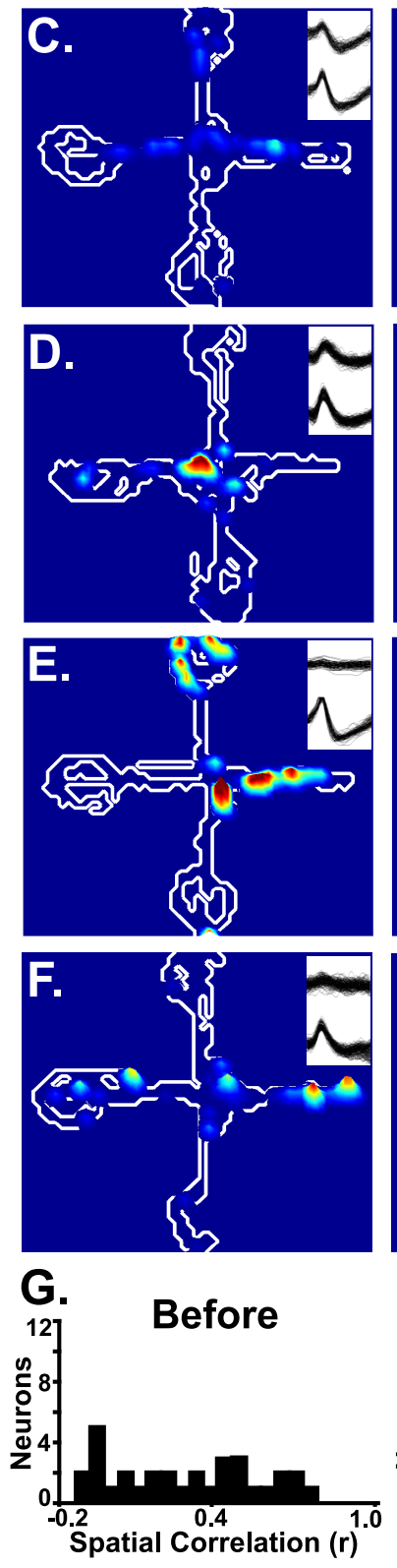

Context B
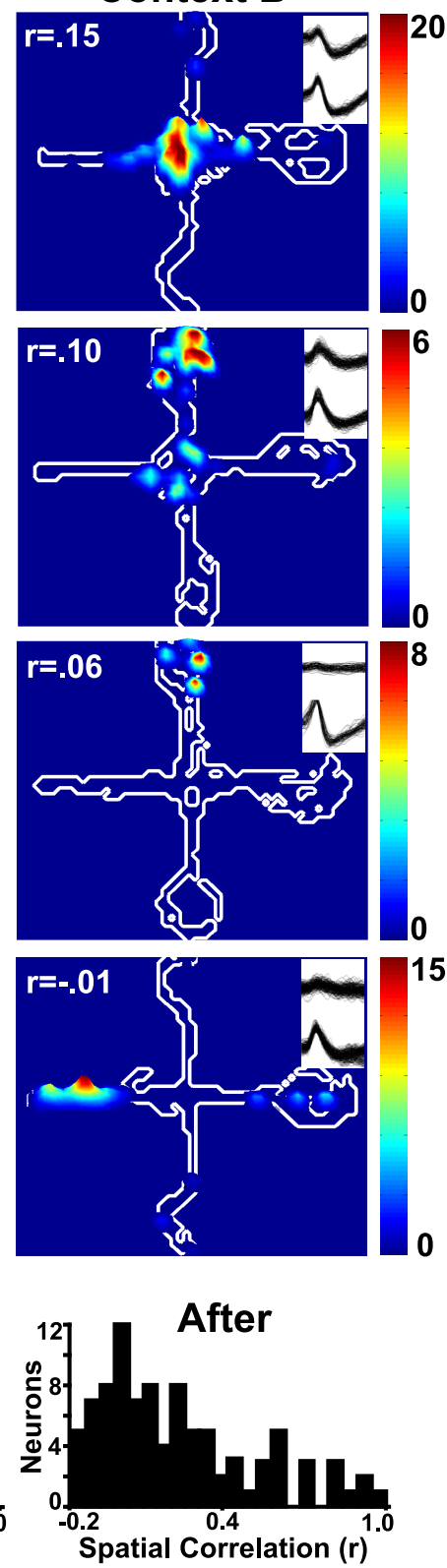

Context A
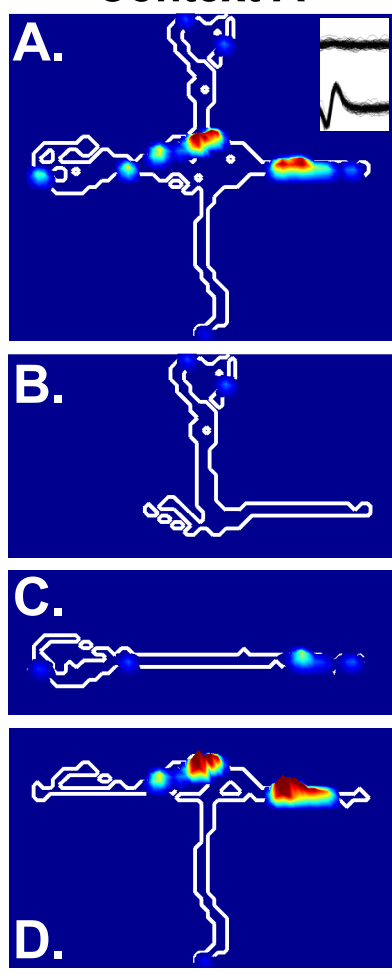

Context B
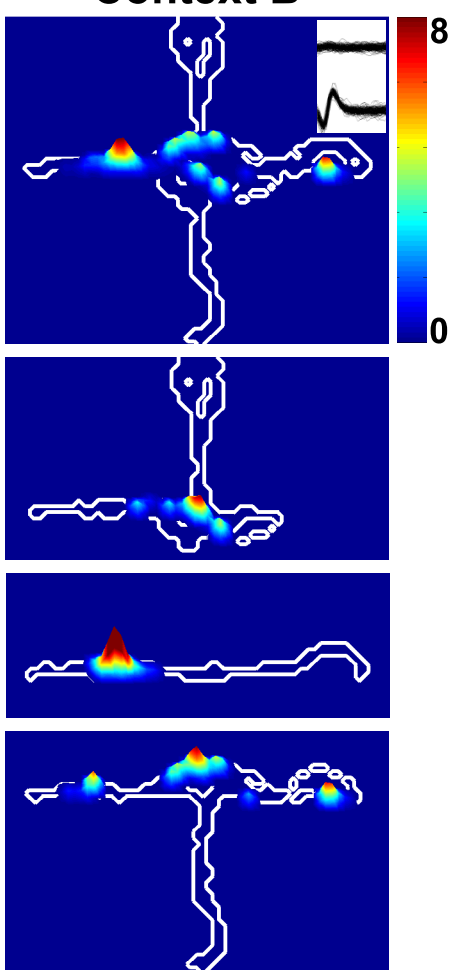

Figure 2. Contour plots illustrating the spatial firing patterns of a neuron that exhibited responses to complex conjunctions of locations and events. The neuron exhibited a place field on the east arm during performance in context $A$ and a field on the west arm in context $B(A)$. The same data are shown in separate firing rate maps for trials that started from the north $(\boldsymbol{B})$, opposite $(\boldsymbol{C})$, and south $(\boldsymbol{D})$ arms. The firing on the east arm in context A occurred primarily on trials that started from the south arm. The firing on the west arm in context B occurred primarily on trials that started on the east arm, opposite the reward.

Many pyramidal neurons exhibited different kinds of responses in the two contexts. Figure 4 illustrates the firing of a neuron that exhibited an event (ITI) response in context $\mathrm{A}$ and a place field in context B. During asymptotic performance, 44 neurons exhibited both spatial- and event-related responses, with one or both being context specific. These results are consistent with previous findings of different kinds of responses when subjects perform different tasks in the same environment (Eichenbaum and Cohen, 1988). The current results indicate that different kinds of responses can emerge even when subjects perform similar behaviors if the contextual demands are different.

Figure 1. Contour plots illustrating the spatial firing patterns during the random reward and asymptotic performance sessions. The regions of the maze visited by the rat are outlined in white. The firing rates are illustrated by the height and color of the contour peaks, with the scale indicated for each neuron. $\boldsymbol{A}$ and $\boldsymbol{B}$ illustrate the firing patterns of two neurons recorded during the first and second halves of the random reward session (Block 1 and Block 2). For each trial, rewards were placed at the end of randomly designated arms, and the rat started at one of the three nonrewarded arms. $\boldsymbol{C}-\boldsymbol{F}$ illustrate the context-specific firing patterns of neurons recorded during asymptotic performance. Each pair of plots illustrates neuronal firing during the first half of the session (Context A), when the reward was always placed on the east arm, and during the second half (Context $B$ ), when the reward was always placed on the west arm. The firing patterns were similar across the two blocks of trials of the random reward session $(\boldsymbol{A}, \boldsymbol{B})$, but they were markedly different in the two blocks of trials (contexts) during asymptotic performance $(\boldsymbol{C}-\boldsymbol{F})$. Spike waveform overlays showing both wires of the stereotrodes recorded during each half session, and pixel by pixel spatial correlation coefficients $(r)$ are given for each pair of plots. $G$ illustrates the distribution of spatial correlation scores for all of the neurons that exhibited place fields before and after learning. 


\section{Context A}
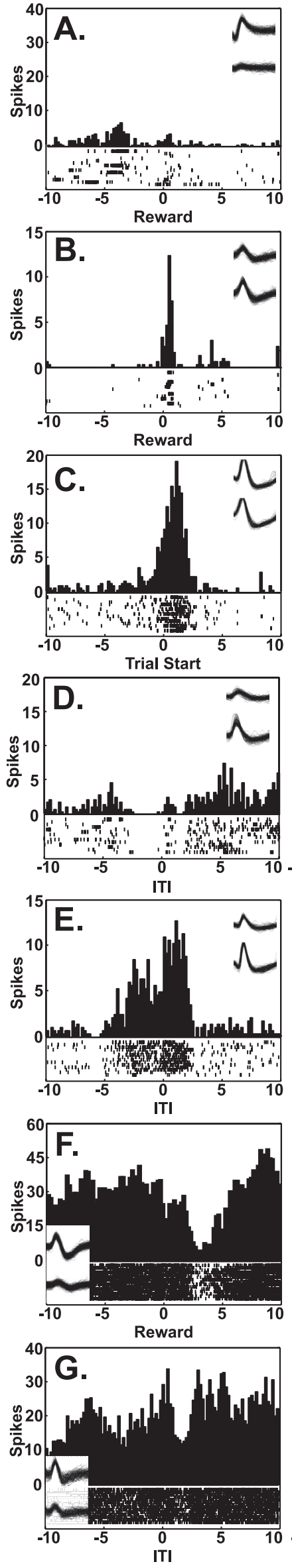

Context B
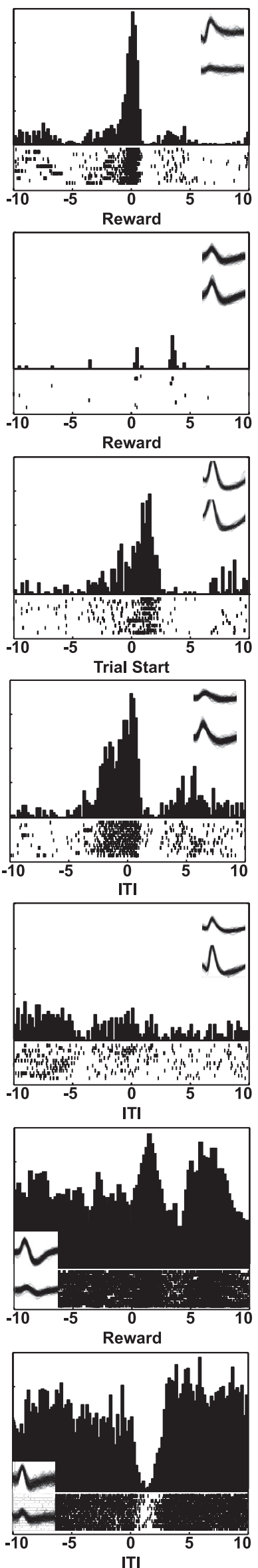

Event-related responses of hippocampal interneurons

Putative hippocampal interneurons also developed contextspecific responses to task-relevant stimuli (Fig. $3 F, G$, Table 1). One hundred twenty putative interneurons were included in the analysis, 44 during the random reward session and 76 during asymptotic performance. The percentage of interneurons exhibiting a context-specific reward response was significantly increased after training $\left(\chi_{(1)}^{2}=7.16 ; p<0.01\right)$. Because interneurons with firing related to the rats' velocity were eliminated from the analysis (see Materials and Methods), the event-related firing of these neurons cannot be accounted for by changes in the rats' locomotion. Interneurons also exhibited context-specific responses at the start of training trials and when subjects were returned to the ITI platform after training trials during asymptotic performance. However, the percentages of neurons with these responses did not change significantly with training.

\section{Spatial and reward response classification}

Neuronal records were inspected to ensure that spatial firing was not mistakenly classified as a reward response and vice versa. Responses were classified as reward responses if they were time locked to the reward and if they did not occur in the same location when there was no reward. Otherwise, they were classified as spatial responses. Post hoc examination of the records of the neurons with reward responses suggested that the responses were correctly classified.

One possible source of misclassification was if the rat passed through a place field, or a spatial region of elevated firing in the case of interneurons (Kubie et al., 1990), that happened to be on the distal part of the goal arm. This would cause firing immediately before the rat's arrival at the reward. However, elevated firing (i.e., $>60 \%$ of the maximum firing rate) on the distal half of the goal arm was observed in only 12 of $78(15 \%)$ cases for pyramidal neurons and 5 of $24(21 \%)$ cases for interneurons.

A second possibility was the presence of a place field, or region of elevated interneuron firing, positioned precisely at the reward location at the end of the arm, which would cause firing immediately on the rat's arrival at the reward location. To address this, neuronal firing at the reward was compared with firing when the rat was in the same location and facing the same direction when there was no reward (i.e., at the start of trials on the same arm in the other context). For example, firing at the time of the reward in context $\mathrm{A}$, when the reward was on the east arm, was compared with firing at the time the rat was placed on the maze at the end of the east arm during the start of trials in context $\mathrm{B}$. The responses were significantly greater at the time of the reward than during

\section{$\leftarrow$}

Figure 3. Perievent time histograms illustrating event-related firing of individual neurons recorded during asymptotic performance. For each plot, the firing was summed across the 15 trials of each training block (context), with raster displays illustrating the trial-by-trial neuronal firing ( 1 row of tick marks per trial). Twenty seconds of data are shown, from $10 \mathrm{~s}$ before to $10 \mathrm{~s}$ after the event. To focus the analysis on firing that was time locked to the event, the analyses were restricted to $1 \mathrm{~s}$ before and after the event. Examples of pyramidal neuron responses at the time of the reward $(\boldsymbol{A}, \boldsymbol{B})$, the start of training trials $(\boldsymbol{C})$, and arrival at the ITI platform after training trials $(\boldsymbol{D}, \boldsymbol{E})$ are shown. Histogram Cillustrates a similar response in the two contexts. In each of the remaining plots, the firing patterns during the first half of the session (Context A), when the reward was always placed on the east arm, were markedly different from firing during the second half (Context B), when the reward was always placed on the west arm. Histograms illustrating interneuron firing at the time of the reward and the ITI are shown in $\boldsymbol{F}$ and $\boldsymbol{G}$. In $\boldsymbol{F}$, the neuron exhibited a significant decrease in firing rate after the reward in context $A$ but a significant increase in context $B$. In $G$, the neuron exhibited a significant decrease in firing rate at the time of the ITI in context $B$ but not in context $A$. 
Table 1. The numbers of neurons (and percentages of the total) that exhibited different kinds of responses are given for pyramidal neurons and interneurons

\begin{tabular}{|c|c|c|c|c|c|c|c|c|}
\hline & Place fields & $\begin{array}{l}\text { Nonspecific } \\
\text { reward responses }\end{array}$ & $\begin{array}{l}\text { Context-specific } \\
\text { reward responses }\end{array}$ & $\begin{array}{l}\text { Nonspecific } \\
\text { ITI responses }\end{array}$ & $\begin{array}{l}\text { Context-specific } \\
\text { ITI responses }\end{array}$ & Multiple responses & None & Total \\
\hline \multicolumn{9}{|l|}{ Pyramidal neurons } \\
\hline Random reward & $34(32.4 \%)$ & $16(15.2 \%)$ & $7(6.7 \%)$ & $22(21.0 \%)$ & $8(7.6 \%)$ & $224 \dagger(21.0 \%)$ & 44 (41.9\%) & 105 \\
\hline Asymptote & $120(39.2 \%)$ & $33(10.8 \%)$ & $68(22.2 \%)^{*}$ & $43(14.1 \%)$ & $59(19.3 \%)^{*}$ & $9820+(32.0 \%)^{*}$ & $101(33.0 \%)$ & 306 \\
\hline \multicolumn{9}{|l|}{ Interneurons } \\
\hline Random reward & & $7(15.9 \%)$ & $4(9.1 \%)$ & $12(27.3 \%)$ & $11(25.0 \%)$ & $6(13.6 \%)$ & $16(36.4 \%)$ & 44 \\
\hline Asymptote & & $23(30.3 \%)$ & $23(30.3 \%)^{*}$ & $25(32.9 \%)$ & $16(21.1 \%)$ & $30(39.5 \%)^{*}$ & $19(25.0 \%)$ & 76 \\
\hline
\end{tabular}

Because the measure of context specificity of the place fields (spatial correlation coefficient) was continuous rather than categorical, neurons were not classified as to whether they exhibited a context-specific place field or not (for distribution of correlation coefficients, see Fig.1G). Event responses and contextual differences in the responses were identified using categorical statistical tests (see Materials and Methods). They could therefore be objectively classified as to whether they exhibited a context-specific response or not, and the numbers of neurons with context-specific and noncontext-specific event responses are tabulated separately. The numbers of neurons and percentages do not sum to the total because some neurons exhibited more than one type of response (Multiple responses). The numbers of pyramidal neurons that exhibited all three kinds of responses (place fields, reward, and ITI responses) are given separately ( $\dagger$ ). The numbers of neurons that did not exhibit any of these responses are also given (None). Percentages of neurons that were significantly different from the random reward session are indicated (*). Trial start responses did not develop with contextual learning, so those responses were not tabulated.

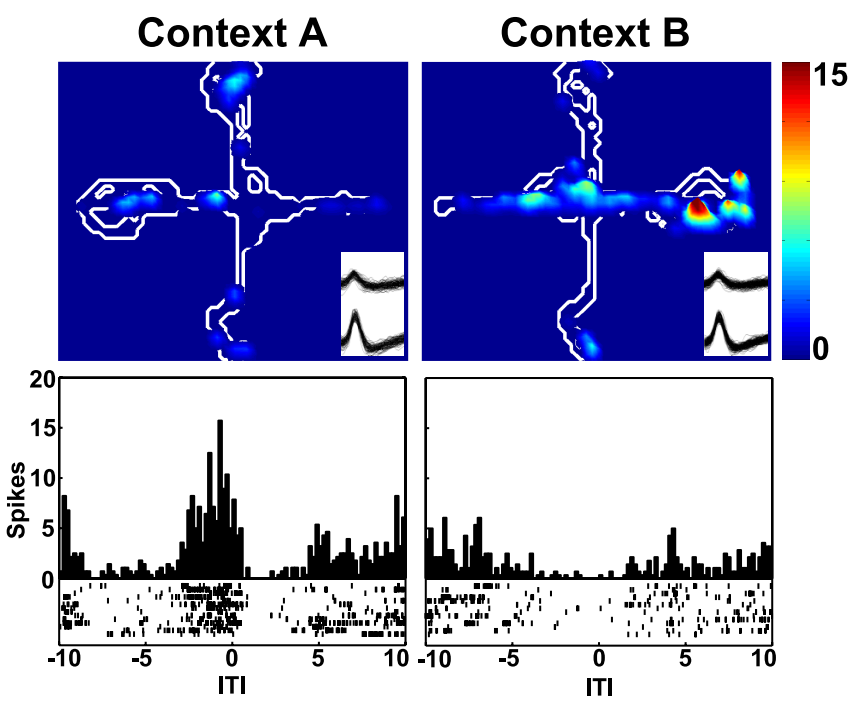

Figure 4. Context-specific spatial-and event-related firing patterns of the same neuron. The neuron exhibited a place field during context $B$ and a response before being returned to the ITI platform during context $\mathrm{A}$.

the start of trials in the same location (pyramidal neurons, $t_{(70)}=$ $3.31, p<0.001$; interneurons, $\left.\left.t_{(29)}=4.12, p<0.001\right]\right)$. Thus, the context-specific reward responses could not have occurred simply because the neurons fired in a spatial location that coincided with the reward location in one context but not the other.

\section{Context-invariant neuronal responses}

Consistent with previous findings (Mizumori et al., 1999), some neurons exhibited response patterns that did not differ in the two contexts (Fig. 3 C). The distribution of the spatial correlation coefficients was heavily skewed toward the lower values after learning (Fig. $1 G$ ), indicating that most of the pyramidal neurons exhibited highly differentiated spatial firing patterns in the two contexts. Nevertheless, some neurons $(n=20 ; 16.7 \%)$ exhibited similar place fields in the two contexts, as indicated by spatial correlation scores between 0.45 and 0.92 . Similarly, some pyramidal neurons exhibited the same reward (10.8\%), ITI (14.1\%), and trial start $(8.5 \%)$ responses in both contexts during asymptotic performance. These percentages did not differ from those observed during the random reward session.

It has been proposed that the hippocampus performs an ongoing comparison of the current context with the memory representation of that context (Mizumori et al., 1999, 2001). This comparative function requires the convergence of information about altered and invariant features of the environment, which is reflected in the neuronal response patterns observed here. In the present study, the majority of the responses were context specific. After learning, $67 \%$ of the neurons with reward responses and $58 \%$ of the neurons with ITI responses exhibited context specificity. Approximately $83 \%$ of the neurons with place fields had spatial correlation scores below 0.45 (Fig. $1 G$ ), suggesting that their responses were also context specific.

\section{Learning-related development of context-specific neuronal responses}

Importantly, context-specific spatial- and event-related responses developed only when the rats were given training in the two contexts. As discussed above, neuronal populations exhibited context-specific responses after training but not during the random reward session. As an additional control, three rats were given two to five additional random reward sessions before beginning context training. These sessions allowed for neuronal recording during repeated training sessions without the context manipulation. Significant block differences in the place fields and event responses did not develop during these sessions. For example, the average spatial correlation coefficients of pyramidal neurons recorded after repeated random reward sessions did not differ from those of neurons recorded during the initial random reward session $\left(F_{(1,28)}=2.06 ; p<0.17\right)$. The percentage of neurons with block-specific reward responses also did not change with repeated random reward sessions $(6.9 \%$ after repeated sessions compared with $6.1 \%$ during the initial session; $\chi_{(1)}^{2}=0.03$; $p<0.34)$. Similarly, the reward responses of interneurons did not develop block specificity with repeated random reward sessions (8.3\% after repeated sessions compared with $9.4 \%$ during the initial session; $\chi_{(1)}^{2}=0.01 ; p<0.92$ ). The number of neurons with block-specific responses to the start of training trials and the ITI were insufficient for analysis.

These results indicated that the development of contextspecific firing patterns could not be attributed to repeated exposure to the training environment or to changes in arousal or motivation over the course of training or during a given training session. Interestingly, a recent study reported changes in place fields after pavlovian fear conditioning (Moita et al., 2004). However, the extreme changes in anxiety or arousal that occur with fear conditioning could not be ruled out as a causative factor in the altered spatial firing.

If the observed neuronal response patterns were related to contextual learning, then the responses should be stable across repeated asymptotic performance sessions. The number of neuronal records with waveforms that were unambiguously the same across successive days was small. Nevertheless, the available data were consistent with the contextual learning interpretation pro- 
Context A
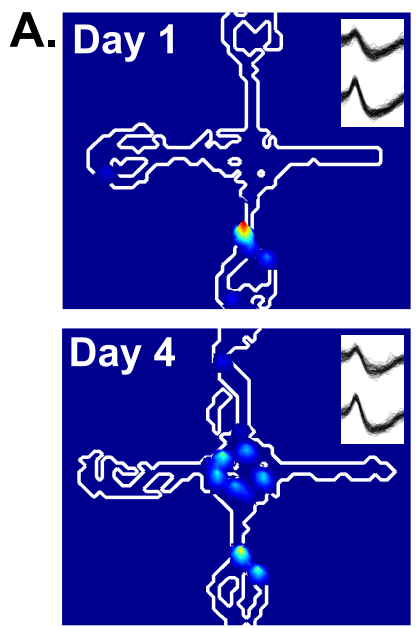

Context A
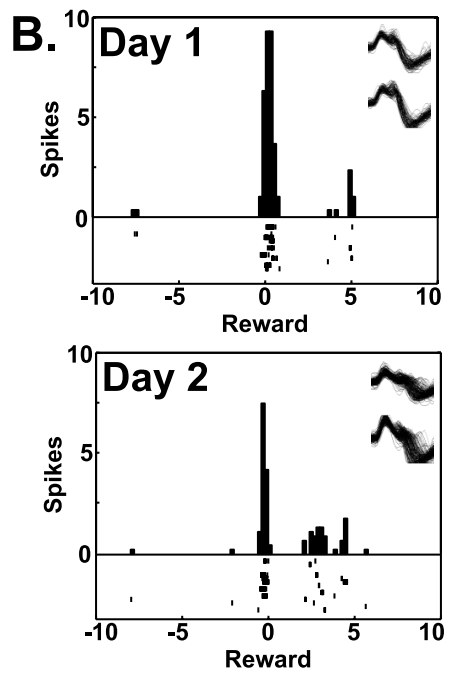

Context A
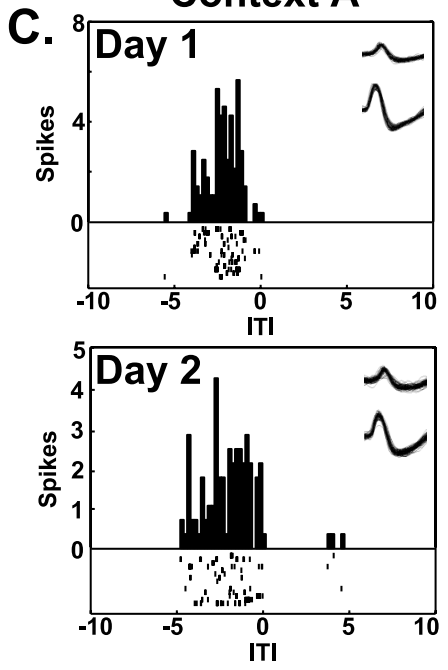
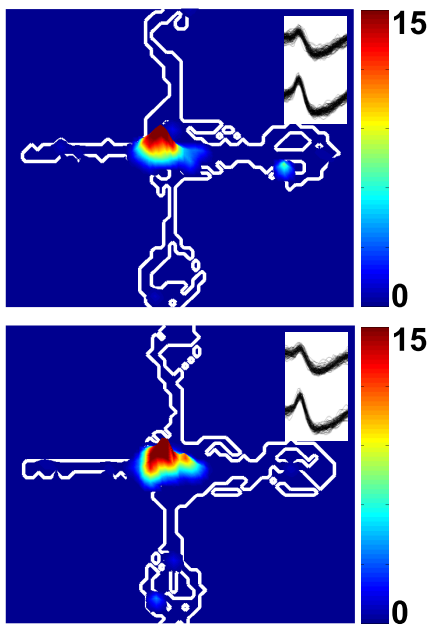

Context B
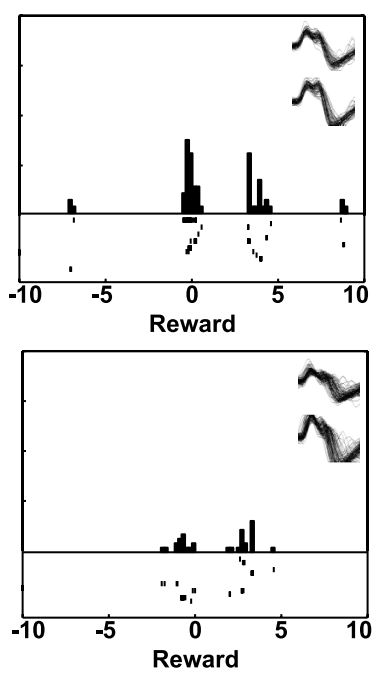

Context B
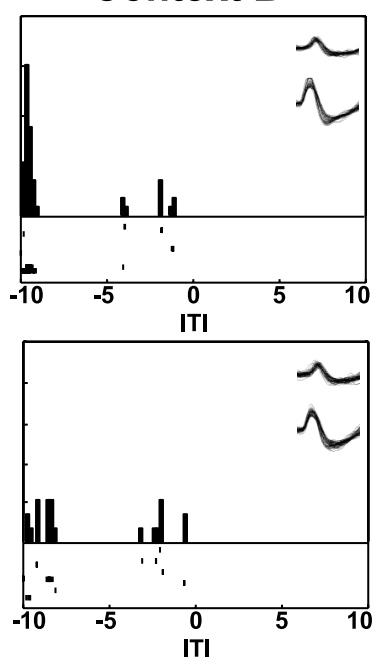

Figure 5. Spatial- and event-related firing across training sessions. The context-specific spatial $(\boldsymbol{A})$, reward $(\boldsymbol{B})$, and ITI $(\boldsymbol{C})$ related firing patterns of three neurons recorded during asymptotic performance are shown. In $\boldsymbol{A}$, context-specific spatial firing patterns were maintained throughout 4 consecutive days of recording. In $B$ and $C$, the reward and ITI responses present in context $A$ were maintained across successive days. The neuron in $\boldsymbol{B}$ did not fire on all 15 of the trials of context $A$ because it fired only when the rat arrived at the reward from the west start arm.

posed here. Figure 5 illustrates three neuronal records that were maintained across multiple days of asymptotic performance. The spatial- and event-related responses of these neurons were quite consistent across sessions.

\section{Discussion}

Hippocampal neurons developed highly differentiated firing patterns when rats learned to discriminate contexts that differed only in terms of the required approach response. Thus, hippocampal context processing is not limited to background stimuli but is also sensitive to the behavioral and mnemonic demands of the task. The context-specific neuronal responses developed only when subjects were exposed to the context manipulation and not in the random reward control condition. Therefore, the differential firing patterns were unambiguously associated with learning about the two contexts and their differing demands. These results support context processing accounts of hippocampal function (Hirsh, 1974; Nadel et al., 1985; Penick and Solomon, 1991; Kim and Fanselow, 1992; Phillips and LeDoux, 1992; Mizumori et al., 1999, 2001; Smith et al., 2004).

The place fields of pyramidal neurons were markedly different in the two contexts. Pyramidal neurons and interneurons also exhibited context-specific responses to task events, including the reward and the subject's return to the ITI platform after training trials. The reward responses may reflect mnemonic processing of the reward and its location, whereas the ITI responses may be related to memory for the reward locations on upcoming (or previous) trials. Neuronal responses to task stimuli have been reported previously (Eichenbaum et al., 1987; Kang and Gabriel, 1998; Moita et al., 2003). The present results suggest that this information is encoded within a framework defined by the context.

Hippocampal interneurons have typically been studied in terms of their contribution to the theta rhythm and their coding of movement information (Feder and Ranck, 1973; Buzsaki et al., 1983). The present results indicate that, like pyramidal neurons, interneurons are highly sensitive to the context. Contextual modulation of interneuron firing could be achieved via direct control by pyramidal neurons (Marshall et al., 2002) or by cortical input (Amaral and Witter, 1989). Interplay between pyramidal neurons and interneurons (Csicsvari et al., 1999; Megias et al., 2001) likely contributes to the development of context-specific neural codes. Therefore, as argued recently (Leutgeb et al., 2000), hippocampal interneurons code more than general movement information and may contribute context-specific information to a larger population code that facilitates context learning.

The functional significance of hippocampal context coding The firing patterns of the hippocampal neuronal population were unique to each context and could therefore serve as neural codes for the context. These context codes are highly sensitive to the spatial geometry of the environment (Muller and Kubie, 1987; O’Keefe and Burgess, 1996; Mizumori et al., 1999). Hippocampal context coding is also sensitive to the nongeometric features of the environment, such as colors or odors (Anderson and Jeffery, 2003; Hayman et al., 2003). The present results extend the idea of hippocampal context coding to incorporate task requirements. Another study found that the problem-solving strategy used by the subjects influences hippocampal coding (Yeshenko et al., 2001). Recently, the hippocampus was implicated in matching behavioral responses to the subjects' motivational state (Kennedy and Shapiro, 2004), suggesting that contexts are also differentiated on the basis of motivational state. The present study manip- 
ulated the reward location, but the previous findings suggest that manipulation of any feature that subjects could use to distinguish the contexts would have produced similar results.

The context processing account of hippocampal function explains why subtle changes in task characteristics are associated with reorganization of the spatial firing patterns, although the environment was unchanged (Markus et al., 1995; Skaggs and McNaughton, 1998; Wood et al., 2000; Yeshenko et al., 2001). That is, subjects perceived the changes in task demands as a shift in the context. This account predicts that hippocampal place fields will undergo reorganization whenever the learning situation changes sufficiently that subjects need to differentiate the new context from the previous. Thus, insofar as hippocampal function is concerned, context can be defined as any situation that has a specific set of behavioral, mnemonic, or strategic demands that must be distinguished from other situations that have different demands.

The distinct context codes observed here could provide a critical means of defeating the interference that occurs when subjects must distinguish similar contexts. One theoretical mechanism for defeating interference that has been attributed to the hippocampus is pattern separation, a process whereby similar inputs lead to highly differentiated representations (McClelland et al., 1995; Gilbert et al., 2001). Consistent with this idea, neurons in the present study produced markedly different responses to the same stimuli depending on the context.

Contextual information plays a critical role in memory retrieval. For example, exposure to the context primes the relevant memories and behavioral responses (Hirsh, 1974; Baddely, 1987; Wickens, 1987). When subjects return to a known context, the hippocampal context code is expressed. The output of the context code could modulate activity in extrahippocampal structures, such as the striatum and the neocortex, so that retrieval of the appropriate behavioral responses and memories is enhanced. For example, the neuron in Figure $1 D$ fired on the north start arm in context B but not in context $\mathrm{A}$. The firing of this neuron and others like it could prime the "right turn" response needed to reach the goal location in context $\mathrm{B}$. When these neurons do not fire, the right turn response may be suppressed, whereas other neurons presumably prime the left turn response appropriate to context A. In this way, the hippocampus could bias the behavioral expression systems of the brain (Mizumori et al., 2004). This idea is supported by recent findings that fornix lesions impaired context cued discrimination learning and disrupted the development of context-specific neuronal response patterns in the cingulate cortex and anterior thalamus (Smith et al., 2004).

\section{Context and spatial navigation}

The present findings raise the question of how the spatial and context processing roles of the hippocampus are related. The spatial layout of an environment is a critical feature of any context, and several authors have used the term "spatial context" to denote this relationship (Nadel et al., 1985; Mizumori et al., 1999; Jeffery et al., 2004). However, the inclusion of spatial information as part of the context may be dictated simply by the ubiquitous nature of spatial information and the fact that the environment typically provides a reliable means of differentiating one context from another. However, the tight coupling of spatial and nonspatial contextual information breaks down when the context is not defined by spatial geometry. The present study was designed so that the spatial layout remained constant while other features of the context were manipulated. Under these circumstances, the hippocampus generated spatial representations that were not controlled exclusively by spatial geometry. Rather, the place fields depended on the context. Thus, spatial coding was subordinate to context coding and is part of a broader context processing function of the hippocampus.

\section{Context and episodic memory}

The hippocampus has been widely implicated in episodic memory (Tulving and Markowitsch, 1998; Aggleton and Brown, 1999; Eichenbaum and Cohen, 2001). Hippocampal neurons respond preferentially to conjunctions of stimuli, such as the cooccurrence of a conditional stimulus and a place (Wood et al., 1999; Moita et al., 2003), and spatial firing can be contingent on past or future actions (Frank et al., 2000; Wood et al., 2000; Ferbinteanu and Shapiro, 2003). Findings such as these suggest that hippocampal neurons encode the relations among stimuli in the interest of episodic memory. Consistent with this idea, some neurons (Fig. 2) responded selectively to the conjunction of a particular place, start position, and goal position (Fig. 5B). Remarkably, even these complex responses depended on the context. Like spatial coding, conjunctive or relational coding was subordinate to context coding.

The idea of context coding is quite compatible with episodic memory accounts of hippocampal function, because episodic memory necessarily involves encoding the context in which events occur. However, the neuronal responses seen here may not be sufficient for the encoding of some types of episodes. For example, because rats readily distinguish one trial from another, such as during alternation learning, they can presumably form distinct episodic memories of individual trials. In the present study, neurons typically responded quite reliably from one trial to the next when subjects entered a place field or experienced an event within a given context (Fig. 3, rasters). In contrast, the neuronal responses were markedly different in the two contexts. Thus, the neuronal responses did not differentiate single-trial episodes as well as they differentiated contexts.

Hippocampal context coding results in a generic representation of a situation that subjects may revisit many times. In contrast, episodic memories explicitly distinguish each visit to a given context as a discrete episode, distinct from all other visits. Because episodic memories incorporate the context in which they occurred, context representations provide a necessary structure for episodic memory. However, these representations are not sufficient to distinguish one experience in a given context from another. Therefore, episodic memory may be mediated by extended circuitry that includes, but is not limited to, the hippocampus. Several authors have suggested that hippocampal memory functions are mediated by circuitry involving the entorhinal cortex, anterior thalamus, prefrontal cortex, and retrosplenial cortex (Aggleton and Brown, 1999; Eichenbaum, 2000; Suzuki and Eichenbaum, 2000; Smith et al., 2004; Wiltgen et al., 2004; Siapas et al., 2005). The present results suggest that the hippocampus contributes contextual information to a wider circuitry for the formation of episodic memories.

\section{References}

Aggleton JP, Brown MW (1999) Episodic memory, amnesia, and the hippocampal-thalamic axis. Behav Brain Sci 22:425-444, discussion $444-489$.

Amaral DG, Witter MP (1989) The three-dimensional organization of the hippocampal formation: a review of anatomical data. Neuroscience 31:571-591.

Anagnostaras SG, Gale GD, Fanselow MS (2001) Hippocampus and contextual fear conditioning: recent controversies and advances. Hippocampus $11: 8-17$. 
Anderson MI, Jeffery KJ (2003) Heterogeneous modulation of place cell firing by changes in context. J Neurosci 23:8827-8835.

Baddely A (1987) Memory and context. In: The oxford companion to the mind (Gregory RL, ed), pp 20-22. Oxford: Oxford UP.

Balaz MA, Capra S, Hartl P, Miller RR (1980) Contextual potentiation of acquired behavior after developing direct context-US associations. Learn Mem 12:383-397.

Blanchard DC, Blanchard RJ (1972) Innate and conditioned reactions to threat in rats with amygdaloid lesions. J Comp Physiol Psychol 81:281-290.

Breese CR, Hampson RE, Deadwyler SA (1989) Hippocampal place cells: stereotypy and plasticity. J Neurosci 9:1097-1111.

Buzsaki G, Leung LW, Vanderwolf CH (1983) Cellular bases of hippocampal EEG in the behaving rat. Brain Res 287:139-171.

Csicsvari J, Hirase H, Czurko A, Mamiya A, Buzsaki G (1999) Fast network oscillations in the hippocampal CA1 region of the behaving rat. J Neurosci 19:RC20(1-4).

Eichenbaum H (2000) A cortical-hippocampal system for declarative memory. Nat Rev Neurosci 1:41-50.

Eichenbaum H, Cohen NJ (1988) Representation in the hippocampus: what do hippocampal neurons code? Trends Neurosci 11:244-248.

Eichenbaum H, Cohen NJ (2001) From conditioning to conscious recollection: memory systems of the brain. New York: Oxford UP.

Eichenbaum H, Kuperstein M, Fagan A, Nagode J (1987) Cue-sampling and goal-approach correlates of hippocampal unit activity in rats performing an odor-discrimination task. J Neurosci 7:716-732.

Fanselow MS (1986) Associative vs topographical accounts of the immediate shock-freezing deficit in rats: implications for the response selection rules governing species-specific defensive reactions. Learn Motiv 17:16-39.

Feder R, Ranck Jr JB (1973) Studies on single neurons in dorsal hippocampal formation and septum in unrestrained rats. II. Hippocampal slow waves and theta cell firing during bar pressing and other behaviors. Exp Neurol 41:532-555.

Ferbinteanu J, Shapiro ML (2003) Prospective and retrospective memory coding in the hippocampus. Neuron 40:1227-1239.

Frank LM, Brown EN, Wilson M (2000) Trajectory encoding in the hippocampus and entorhinal cortex. Neuron 27:169-178.

Freeman Jr JH, Weible A, Rossi J, Gabriel M (1997) Lesions of the entorhinal cortex disrupt behavioral and neuronal responses to context change during extinction of discriminative avoidance behavior. Exp Brain Res 115:445-457.

Gilbert PE, Kesner RP, Lee I (2001) Dissociating hippocampal subregions: a double dissociation between dentate gyrus and CA1. Hippocampus 11:626-636.

Godden D, Baddely A (1975) Context-dependent memory in two natural environments: on land and underwater. Br J Psychol 66:325-331.

Hayman RM, Chakraborty S, Anderson MI, Jeffery KJ (2003) Contextspecific acquisition of location discrimination by hippocampal place cells. Eur J Neurosci 18:2825-2834.

Hirsh R (1974) The hippocampus and contextual retrieval of information from memory: a theory. Behav Biol 12:421-444.

Hollup SA, Molden S, Donnett JG, Moser MB, Moser EI (2001) Accumulation of hippocampal place fields at the goal location in an annular watermaze task. J Neurosci 21:1635-1644.

Jeffery KJ, Anderson MI, Hayman R, Chakraborty S (2004) A proposed architecture for the neural representation of spatial context. Neurosci Biobehav Rev 28:201-218.

Kang E, Gabriel M (1998) Hippocampal modulation of cingulo-thalamic neuronal activity and discriminative avoidance learning in rabbits. Hippocampus 8:491-510.

Kennedy PJ, Shapiro ML (2004) Retrieving memories via internal context requires the hippocampus. J Neurosci 24:6979-6985.

Kim JJ, Fanselow MS (1992) Modality-specific retrograde amnesia of fear. Science 256:675-677.

Kobayashi T, Tran AH, Nishijo H, Ono T, Matsumoto G (2003) Contribution of hippocampal place cell activity to learning and formation of goaldirected navigation in rats. Neuroscience 117:1025-1035.

Kubie JL, Muller RU, Bostock E (1990) Spatial firing properties of hippocampal theta cells. J Neurosci 10:1110-1123.

Leutgeb S, Ragozzino KE, Mizumori SJ (2000) Convergence of head direc- tion and place information in the CA1 region of hippocampus. Neuroscience 100:11-19.

Maren S (2001) Neurobiology of Pavlovian fear conditioning. Annu Rev Neurosci 24:897-931.

Markus EJ, Qin YL, Leonard B, Skaggs WE, McNaughton BL, Barnes CA (1995) Interactions between location and task affect the spatial and directional firing of hippocampal neurons. J Neurosci 15:7079-7094.

Marshall L, Henze DA, Hirase H, Leinekugel X, Dragoi G, Buzsaki G (2002) Hippocampal pyramidal cell-interneuron spike transmission is frequency dependent and responsible for place modulation of interneuron discharge. J Neurosci 22:RC197(1-5).

McClelland JL, McNaughton BL, O’Reilly RC (1995) Why there are complementary learning systems in the hippocampus and neocortex: insights from the successes and failures of connectionist models of learning and memory. Psychol Rev 102:419-457.

McNaughton BL, O'Keefe J, Barnes CA (1983a) The stereotrode: a new technique for simultaneous isolation of several single units in the central nervous system from multiple unit records. J Neurosci Methods 8:391-397.

McNaughton BL, Barnes CA, O'Keefe J (1983b) The contributions of position, direction, and velocity to single unit activity in the hippocampus of freely-moving rats. Exp Brain Res 52:41-49.

Megias M, Emri Z, Freund TF, Gulyas AI (2001) Total number and distribution of inhibitory and excitatory synapses on hippocampal CA1 pyramidal cells. Neuroscience 102:527-540.

Mizumori SJ, Ragozzino KE, Cooper BG, Leutgeb S (1999) Hippocampal representational organization and spatial context. Hippocampus 9:444-451.

Mizumori SJ, Cooper BG, Leutgeb S, Pratt WE (2001) A neural systems analysis of adaptive navigation. Mol Neurobiol 21:57-82.

Mizumori SJ, Yeshenko O, Gill KM, Davis DM (2004) Parallel processing across neural systems: implications for a multiple memory system hypothesis. Neurobiol Learning Mem 82:278-298.

Moita AP, Svetlana R, Zhou Y, LeDoux JE, Blair HT (2003) Hippocampal place cells acquire location-specific response to the conditioned stimulus during auditory fear conditioning. Neuron 37:485-497.

Moita AP, Rosis S, Zhou Y, LeDoux JE, Blair HT (2004) Putting fear in its place: remapping of hippocampal place cells during fear conditioning. J Neurosci 24:7015-7023.

Muller RU, Kubie JL (1987) The effects of changes in the environment on the spatial firing of hippocampal complex-spike cells. J Neurosci 7:1951-1968.

Myers CE, Gluck M (1994) Context, conditioning, and hippocampal rerepresentation in animal learning. Behav Neurosci 108:835-847.

Nadel L, Willner J, Kurz EM (1985) Cognitive maps and environmental context. In: Context and learning (Balsam P, Tomie A, eds), pp 385-406. Hillsdale, NJ: Erlbaum.

O'Keefe J, Burgess N (1996) Geometric determinants of the place fields of hippocampal neurons. Nature 381:425-428.

O'Keefe J, Dostrovsky J (1971) The hippocampus as a spatial map. Preliminary evidence from unit activity in the freely-moving rat. Brain Res 34:171-175.

Penick S, Solomon PR (1991) Hippocampus, context, and conditioning. Behav Neurosci 105:611-617.

Phillips RG, LeDoux JE (1992) Differential contribution of amygdala and hippocampus to cued and contextual fear conditioning. Behav Neurosci 106:274-285.

Siapas AG, Lubenov EV, Wilson MA (2005) Prefrontal phase locking to hippocampal theta oscillations. Neuron 46:141-151.

Skaggs WE, McNaughton BL (1998) Spatial firing properties of hippocampal CA1 populations in an environment containing two visually identical regions. J Neurosci 18:8455-8466.

Smith DM, Wakeman D, Patel J, Gabriel M (2004) Fornix lesions impair context-related cingulothalamic neuronal patterns and concurrent discrimination learning. Behav Neurosci 118:1225-1239.

Song EY, Kim YB, Kim YH, Jung MW (2005) Role of active movement in place-specific firing of hippocampal neurons. Hippocampus 15:8-17.

Speakman A, O'Keefe J (1990) Hippocampal complex spike cells do not change their place fields if the goal is moved within a cue controlled environment. Eur J Neurosci 2:544-555. 
Suzuki WA, Eichenbaum H (2000) The neurophysiology of memory. Ann NY Acad Sci 911:175-191.

Tulving E, Markowitsch HJ (1998) Episodic and declarative memory: role of the hippocampus. Hippocampus 8:198-204.

Wickens DD (1987) The dual meanings of context: implications for research, theory, and applications. In: Memory and learning: the Ebbinghaus Centennial Conference (Gorfin DS, Hoffman RR, eds), pp 135-152. Hillsdale, NJ: Erlbaum.

Wiebe SP, Staubli UV (2001) Recognition memory correlates of hippocampal theta cells. J Neurosci 21:3955-3967.

Wiltgen BJ, Brown RA, Talton LE, Silva AJ (2004) New circuits for old memories: the role of the neocortex in consolidation. Neuron 44:101-108.
Wood ER, Dudchenko PA, Eichenbaum H (1999) The global record of memory in hippocampal neuronal activity. Nature 397:613-616.

Wood ER, Dudchenko PA, Robitsek RJ, Eichenbaum H (2000) Hippocampal neurons encode information about different types of memory episodes occurring in the same location. Neuron 27:623-633.

Yeshenko O, Guazzelli A, Mizumori SJY (2001) Differential hippocampal neuronal activity during place or response performance on a t-maze. Soc Neurosci Abstr 27:953.8.

Yeshenko O, Guazzelli A, Mizumori SJ (2004) Context-dependent reorganization of spatial and movement representations by simultaneously recorded hippocampal and striatal neurons during performance of allocentric and egocentric tasks. Behav Neurosci 118:751-769. 